\title{
Synthesis of Copper-nitrogen Codoped Carbon Quantum Dots Using Frangipani as a Carbon Source and Application of Metronidazole Determination
}

Lan Yuwei Lan ( $\square$ lanyuwei78@163.com )

Guangxi University https://orcid.org/0000-0002-8413-6401

Yuwei Lan

Guangxi University

Wenbin Bao

Guangxi University

Chunfeng Liang

Guangxi University

Guowei Li

Guangxi University

Jiachu Yang

Guangxi University

Liubai Wei

Guangxi University

Qionghua Su

Guangxi University

\section{Research Article}

Keywords: Cu/NCQDs, fluorescence, metronidazole, fluorescence probe

Posted Date: December 14th, 2021

DOI: https://doi.org/10.21203/rs.3.rs-1030469/v1

License: (c) (i) This work is licensed under a Creative Commons Attribution 4.0 International License.

Read Full License 


\section{Abstract}

High-fluorescence $\mathrm{Cu} / \mathrm{N}$ codoped carbon quantum dots (Cu/NCQDs) were prepared by a one-step hydrothermal method using frangipani as the carbon source and copper acetate as the copper source. The Cu/NCQDs exhibited high-intensity, stable blue fluorescence that is independent of the excitation wavelength. Since metronidazole can effectively quench the fluorescence intensity of Cu/NCQDs, a metronidazole fluorescence-detection method using Cu/NCQDs as the fluorescence probe was developed, and the quenching mechanism was studied.The method has the advantages of simplicity, speed, and low cost. Besides, it has a wider linear range and detection limit. Further, the metronidazole content in actual samples was determined by this method, with good results.

\section{Statement Of Novelty}

Carbon quantum dots (QDS) are fluorescent carbon nanomaterials with dual advantages of both fluorescent materials and nanomaterials, so they have been widely studied. In recent years, developing the concept of "green chemistry" to synthesize green carbon quantum dots has become a research hotspot, and more green carbon materials and simple and efficient synthesis methods need to be developed. Meanwhile, heteroatomic doped carbon quantum dots (QDS) have attracted much attention because of their novel optical properties, which are different from pure carbon dots. There are many researches on the doping of non-metallic elements with carbon quantum dots, but there are few reports on the doping of metallic elements, especially transition metal elements, and there is a great space for development. Based on the above research background, a one-step hydrothermal process was used to process natural biomass to obtain transition metal codoped carbon quantum dots.

\section{Introduction}

Metronidazole (2-methyl-5-nitro-1H-imidazol-1-ethanol, MNZ), a 5-nitroimidazole antibiotic, is mainly used to treat or prevent systemic or local infectious diseases caused by anaerobic bacteria and protozoa, such as lower respiratory, female genital tract, and digestive tract infections. In animal husbandry, metronidazole is often used as a feed or drug additive to promote the growth of pigs, cattle, and sheep and to inhibit the growth of parasites [1-3]. However, studies have shown that metronidazole has teratogenic effects on animal and plant cells, including carcinogenic and mutagenic effects. If used irrationally or abused in livestock and poultry feed, metronidazole residues may persist in animal tissue and could be transmitted through the food chain, thereby indirectly causing damage to the human body, such as epileptic neuropathy $[4,5]$. As is well known, the abuse of antibiotics, release of antibiotics into the environment, and development of drug-resistant genes in natural ecosystems are increasingly serious global threats. Therefore, the establishment of a rapid and effective metronidazole-detection method is of significant importance in ensuring food safety.

Thus far, high-performance liquid chromatography [6, 7], ultra-high-performance liquid chromatographytandem mass spectrometry [8-10], electrochemical method [11, 12], chemiluminescence [13], and other 
analytical methods have been widely employed to detect metronidazole. In particular, the fluorescencedetection method based on carbon quantum dots (CQDs) is promising because of its low cost and simple operation.

CQDs are novel carbon nanomaterials that exhibit photoluminescence $(\mathrm{PL})$ and fluorescence properties. However, pure CQDs, because of the few functional groups on their surfaces, exhibit a low quantum yield (QY), which limits their practical application [14]. Studies have shown that surface functionalization and chemical heteroatomic doping are effective methods for improving the fluorescence properties of CQDs and expanding their application range [15]. In this regard, $\mathrm{N}, \mathrm{P}, \mathrm{S}, \mathrm{B}, \mathrm{Cl}$, and other non-metallic heteroatoms have been extensively employed as dopants for the single doping or co-doping of CQDs. In particular, when CQDs are doped with $\mathrm{N}$, whose atomic radius and valence electron structure are similar to those of $C$, the surface of the CQDs becomes positively charged, which significantly improves not only the interaction between the target analyte and CQDs, but also the QY of the CQDs. Resultantly, many studies on the heteroatom doping of CQDs have employed the $\mathrm{N}$ atom as the dopant [16-18]. However, compared with nonmetal impurity atoms, metal ions, particularly transition metal ions, have more volatile electronics and do not occupy orbits; further, they have larger atomic radii than non-metallic atoms [19]. Thus, transition metal atoms can change the surface charge density of CQDs, which is more advantageous [20]. In recent years, the research on the metal-atom doping of CQDs has developed rapidly. Metal atoms, including $\mathrm{Cu}, \mathrm{Fe}, \mathrm{Zn}$, and $\mathrm{Mn}$, have been extensively utilized. Among them, $\mathrm{Cu}$ is widely employed in the doping of CQDs because of its safety; additionally, it facilitates the interaction between aminocarboxyl and hydroxyl groups on the surfaces of CQDs [21]. Moreover, the Cu atoms doped on the surface of CQDs can form coordination complexes with most small molecules, i.e., chelation, which is essential for the detection of analytes in the environment.

Therefore, high-fluorescence $\mathrm{Cu} / \mathrm{N}$ co-doped carbon quantum dots (Cu/NCQDs) were prepared by onestep hydrothermal method using fractionis as carbon source and copper acetate as copper source. $\mathrm{Cu} / \mathrm{NCQDs}$ showed strong blue light and excitation independent fluorescence properties. Since metronidazole can effectively quench the fluorescence intensity of Cu/NCQDs, a metronidazole fluorescence-detection method using Cu/NCQDs as the fluorescence probe was developed, and the quenching mechanism was studied. The metronidazole content in actual samples was detected by this method, and good results were obtained.

\section{Experimental}

\subsection{Experimental drugs}

Frangipani, ethylenediamine( $\mathrm{C} 2 \mathrm{H} 8 \mathrm{~N} 2)$, acetone $\square \mathrm{C} 3 \mathrm{H} 6 \mathrm{O} \otimes$, copper acetate $\square \mathrm{Cu}(\mathrm{CH} 3 \mathrm{COO}) 2 \cdot \mathrm{H} 2 \mathrm{O})$, Metronidazole(C6H9N3O3), citric acid (C6H8C6), monopotassium phosphate(NaH2PO4), potassium chloride(KCl), glucose (C6H12O6), ascorbic acid(C6H8O6), saccharose (C12H22O11), beta cyclodextrin(C42H70035), L-cysteine(C3H7NO2S), magnesium nitrate(Mg (NO3)2), ammonium chloride $(\mathrm{NH} 4 \mathrm{Cl} \rrbracket$, calcium chloride $\square \mathrm{CaCl} 2 \rrbracket$, sodium carbonate $\square \mathrm{Na} 2 \mathrm{CO} \rrbracket$. 
All drugs were analytically pure, and distilled water was used in the experiment without further purification.

\subsection{Test and characterization instruments}

electro- thermostatic blast oven(DHG-9146A), UV-VIS spectrophotometer】UV-1800囚, fluorospectro photometer[RF-5301》, TEMITECNAL G2 F30区,

Fourier Infrared Spectrometer(Nicolet iS50), XPS口ESCALAB 250Xi囚, Fluorescence Lifetime Spectrometer $\square$ Fs980冈, centrifuge $\amalg \mathrm{H} 1850 \rrbracket$.

\subsection{Preparation of $\mathrm{Cu} / \mathrm{NCQDs}$}

The specific preparation method of CQDs is as follows: $1.0 \mathrm{~g}$ of frangipani powder was dispersed in 40 $\mathrm{mL}$ of deionized water. Subsequently, $0.15 \mathrm{~mL}$ of ethylenediamine $(20 \mathrm{mmol} / \mathrm{L})$ was added as the nitrogen source. After ultrasonic treatment for $10 \mathrm{~min}$, the mixed solution was transferred into a $50 \mathrm{~mL}$ high-pressure reaction kettle and placed in an electric thermostatic air blow-drying oven at the temperature of $200^{\circ} \mathrm{C}$. Thereafter, the reaction kettle was cooled naturally to room temperature, and the reaction solution was pumped and filtered. The filtrate was centrifuged at $4000 \mathrm{rpm}$ for $10 \mathrm{~min}$, and the supernatant was filtered again using a water-soluble filter membrane (pore size: $0.22 \mu \mathrm{m}$ ) to obtain the purified fluorescent $\mathrm{Cu} / \mathrm{NCQD}$ solution. The prepared CQD solution was stored in four refrigerators for later use.

Using the same preparation process, except with the addition of copper acetate monohydrate, N-doped CQDs (NCQDs) were prepared as contrast materials.

\subsection{Fluorescence detection of metronidazole}

The Cu/NCQD standard metronidazole (MNZ) solutions ( $100 \mu \mathrm{L}$ portions) of different concentrations were successively poured into a series of $10 \mathrm{~mL}$-colorimetric tubes and diluted to scale using a citric acidsodium dihydrogen phosphate buffer ( $\mathrm{pH}=4$; Conc.: $0.2 \mathrm{~mol} / \mathrm{L})$. After shaking, the metronidazole solution was stable for $3 \mathrm{~min}$. The fluorescence spectrum was recorded with a 1-cm quartz colorimeter, using an RF-5301 PC fluorescence spectrophotometer. The fluorescence intensities with and without the MNZ solution were denoted as $F$ and $F_{0}$, respectively. The excitation wavelength was set at $355 \mathrm{~nm}$, and the excitation and emission slit widths were both $3 \mathrm{~nm}$.

\subsection{Pretreatment of honey samples}

Metronidazole tablets ( $0.2 \mathrm{~g}$ each) and honey were purchased from Ali Health Pharmacy(Nanning,China). Each metronidazole tablet was ground into powder using an agate mortar, dissolved in distilled water, ultrasonicated for $15 \mathrm{~min}$, and dissolved in a 100-mL volumetric bottle for subsequent analysis. The specific method for pretreating the honey was obtained from the literature [11], as follows: $10 \mathrm{mg}$ of the honey sample was dissolved in $1 \mathrm{~mL}$ of an acetone solution (5\%). After $15 \mathrm{~min}$ of ultrasonic treatment, 1 
$\mathrm{mL}$ of the sample solution was poured into a $10 \mathrm{~mL}$-colorimetric tube, and $100 \mu \mathrm{L}$ of the $\mathrm{Cu} / \mathrm{NCQD}$ solution was added. Afterward, the solution was diluted to scale with the citric acid-sodium dihydrogen phosphate buffer $(\mathrm{pH}=4)$.

\subsection{Determination of the metronidazole working curve}

$\mathrm{Cu} / \mathrm{NCQD}(100 \mu \mathrm{L})$ and metronidazole solutions $(100 \mu \mathrm{L})$ of different concentrations were added separately to a series of $10 \mathrm{~mL}$-colorimetric tubes and diluted to scale with the citric acid-sodium dihydrogen phosphate buffer $(\mathrm{pH}=4)$. Thereafter, the solution was shaken and left for 5 mins; afterward, $F_{0}$ and $F$ were recorded using the fluorescence spectrometer, and the fluorescence intensity without and with metronidazole was determined using the formula: $F=F_{0}-F$, as shown in Figure 2. Figure 2-A shows the fluorescence spectra of the Cu/NCQD solution with metronidazole in different concentrations, and Figure 2-B shows the corresponding correction curves. The results show that with an increase in the metronidazole concentration in the range of $0-350 \mathrm{~mol} / \mathrm{L}$, the fluorescence intensity of the $\mathrm{Cu} / \mathrm{NCQDs}$ gradually decreases. The linear equation was $F=-3.235+9.064 \mathrm{CMNZ}(\mu \mathrm{mol} / \mathrm{L})$, the correlation coefficient $\left(R^{2}\right)$ was 0.996 , and the detection limit was 0.28 , calculated by the three standard deviation method $(3 / S)(\mu \mathrm{mol} / L)$, where is the standard deviation of the blank signal $(n=11)$, and $S$ is the slope of the linear calibration curve.

\section{Results And Discussion}

\subsection{Morphology and structure characterization of the Cu/NCQDs}

The morphology and size of the Cu/NCQDs were determined by transmission electron microscopy. The results are shown in Figures 3. As shown in Figure 3-A, the prepared Cu/NCQDs exhibited a nearly spherical morphology and good dispersion in water. Figure 3-B shows the particle-size distribution of the $\mathrm{Cu} / \mathrm{NCQDs}$. Statistically, the particle-size range of Cu/NCQDs is $1.90-3.70 \mathrm{~nm}$, and the Gaussian fitting curve shows that the average particle size is $2.58 \mathrm{~nm}$.

Figure 3-C shows the Fourier transform infrared (FTIR) spectra of the NCQDs and Cu/NCQDs, which are exploited to characterize the functional groups on the surface of the CQDs. In Figure 3-C, a wide absorption band can be observed near $3428 \mathrm{~cm}^{-1}$, and the $-\mathrm{NH} / \mathrm{OH}$ stretching vibration of the Cu/NCQDs [21].The absorption peaks slightly lower than $3000 \mathrm{~cm}^{-1}$ and $1384 \mathrm{~cm}^{-1}$ correspond to the stretching vibration and bending vibration of the alkyl $\mathrm{C}-\mathrm{H}$ bond, respectively, and the peak near $1710 \mathrm{~cm}^{-1}$ is related to the stretching vibration of the $-\mathrm{COOH}$ group. The absorption peaks at 1000 and $1217 \mathrm{~cm}^{-1}$ correspond to the symmetric and asymmetric stretching vibrations of carbon and oxygen compounds (C-O-C), respectively [21]. The above results indicate the abundance of hydrophilic groups $\left(-\mathrm{OH}, \mathrm{NH}_{2}\right.$, and $-\mathrm{COOH}$ ) on the surface of the $\mathrm{Cu} / \mathrm{NCQDs}$, which explains their good water solubility. Further, the hydrophilic groups facilitate the chelation of $\mathrm{Cu} / \mathrm{NCQDs}$ with $\mathrm{Cu}^{2+}$, as well as promote the surface passivation process of the CQDs and Cu doping. Notably, the $870-1000 \mathrm{~cm}^{-1}$ range is denoted as the metal-sensitive region $[33,21,22]$. Different from the NCQDs, the Cu/NCQDs exhibited partial absorption 
in the metal-sensitive region. This is related to the stretching vibration of the $\mathrm{N}-\mathrm{Cu}-\mathrm{N}$ bond and confirms the formation of a Cu coordination complex in the Cu/NCQDs. The FT-IR spectra showed that $\mathrm{N}$ and $\mathrm{Cu}$ were successfully doped on the surface or the Cu/NCQDs in the form of related groups.

X-ray photoelectron spectroscopy (XPS) was conducted to further confirm the structural composition of the $\mathrm{Cu} / \mathrm{NCQDs}$. In the XPS full-scan spectra of the Cu/NCQDs (Figure 3-D), four peaks are observed at binding energies of $284.82,399.98,531.32$, and $932.66 \mathrm{eV}$, belonging to $\mathrm{C} 1 \mathrm{~s}, \mathrm{~N} 1 \mathrm{~s}, \mathrm{O} 1 \mathrm{~s}$, and $\mathrm{Cu} 2 \mathrm{p}$, respectively. The $C 1$ s high-resolution spectrum (Fig. 4-A) shows four peaks at 284.55, 285.94, 287.11, and $288.10 \mathrm{eV}$, indicating the existence of carbon in four different states: $\mathrm{C}-\mathrm{C} / \mathrm{C}=\mathrm{C}, \mathrm{C}-\mathrm{N} / \mathrm{C}-\mathrm{O}, \mathrm{C}=\mathrm{O}$, and COO- [15], respectively. Figure 4-B shows the high-resolution spectrum of $01 \mathrm{~s}$, and the peaks at 530.99 $\mathrm{eV}$ and $532.09 \mathrm{eV}$ correspond to the $\mathrm{C}=\mathrm{O}$ and $\mathrm{C}-\mathrm{OH} / \mathrm{C}-\mathrm{O}-\mathrm{C}$ bonds $[23,34]$, respectively. The $\mathrm{N} 1 \mathrm{~s}$ highresolution spectrum (Fig. 4-C) confirmed the presence of C-N-C (399.72 eV), N-C3 (400.59 eV), and N$\mathrm{H}$ bonds (401.87 eV) [35, 22]. The Cu 2p spectra (Fig. 4-D) show two peaks at $933.0 \mathrm{eV}$ and $952.50 \mathrm{eV}$, attributable to $\mathrm{Cu} 2 \mathrm{p} 2 / 3$ and $\mathrm{Cu} 2 \mathrm{p} 1 / 3$, respectively, indicative of the $\mathrm{N}-\mathrm{Cu}-\mathrm{N}$ covalent bond in porphyrin-metal complexes. In conclusion, the XPS results are consistent with the FT-IR spectroscopy results, which proves that $\mathrm{Cu}$ and $\mathrm{N}$ were successfully codoped in the $\mathrm{Cu} / \mathrm{NCQD}$ skeleton.

\subsection{Study on fluorescence properties of Cu/NCQDs}

To explore the fluorescence performance of the Cu/NCQDs, ultraviolet-visible (UV-vis) and fluorescence spectroscopies were performed. Figure 5-A shows the UV-vis absorption spectra of the Cu/NCQDs, as well as their fluorescence excitation and emission spectra. The maximum excitation and emission wavelengths of the Cu/NCQDs are approximately $355 \mathrm{~nm}$ and $443 \mathrm{~nm}$, respectively. As illustrated in Figure S3, the Cu/NCQDs exhibit a pale-yellow color in sunlight. The bright blue fluorescence under 360 $\mathrm{nm}$ UV light indicates that the Cu/NCQDs have unique optical properties.

Figure 5-B shows the fluorescence emission spectra of the Cu/NCQDs at different excitation wavelengths. Noticeably, the fluorescence intensity increases gradually as the excitation wavelength increases from $300 \mathrm{~nm}$ to $355 \mathrm{~nm}$. Therefore, $355 \mathrm{~nm}$ was adopted as the best excitation wavelength in subsequent experiments. Notably, with varying the excitation wavelengths, the maximum emission wavelength of the $\mathrm{Cu} / \mathrm{NCQDs}$ remained $443 \mathrm{~nm}$ without any noticeable red or blue shift. This result shows that the watersoluble $\mathrm{Cu} / \mathrm{NCQDs}$ exhibit PL behavior regardless of the excitation wavelength, which is in contrast to most reported CQDs, whose PL behaviors depend on the excitation wavelength. This PL behavior of the $\mathrm{Cu} /$ NCQDs is attributed to its narrow particle-size distribution, as well as its highly uniform surface structure and surface-energy trapping ability $[24,25]$. Notably, the emission peak of the Cu/NCQDs at 443 $\mathrm{nm}$ is independent of the excitation wavelength, which reduces the fluorescence interference from the CQDs themselves in the detection process of the target. This evidences the application potential of $\mathrm{Cu} /$ NCQDs in the analysis and detection of analytes.

3.3 Study of the factors influencing the fluorescence performance of Cu/NCQDs 
A series of experiments was conducted to investigate the factors affecting the fluorescence performance of the $\mathrm{Cu} / \mathrm{NCQDs}$. The experimental results are shown in Figure 6-A. In salt solutions of different concentrations, the fluorescence intensity of the Cu/NCQDs remained almost unchanged, indicating that they are not affected by the solution ionic strength and have excellent salt tolerance. Therefore, $\mathrm{Cu} / \mathrm{NCQDs}$ can be applied in biochemical analyses. Furthermore, as shown in Figure 6-B, the fluorescence intensity of the $\mathrm{Cu} / \mathrm{NCQD}$ s is relatively strong and stable in acidic environments. With the increase in the $\mathrm{pH}$, the fluorescence intensity decreases significantly. This phenomenon may be related to the $\mathrm{Cu}$ ions on the surface of the $\mathrm{Cu} / \mathrm{NCQDS}$, which inhibit hydrolysis in an acidic environment and promote hydrolysis in an alkaline environment. Thus, the content of $\mathrm{Cu}$ ions on the quantum dots decreases under alkaline conditions, which decreases the fluorescence intensity. Moreover, several studies have proven the relationship between the fluorescence intensity and doped $\mathrm{Cu}$ ions. In addition, the emission wavelength of the $\mathrm{Cu} / \mathrm{NCQDs}$ does not vary with $\mathrm{pH}$, indicating that the prepared $\mathrm{Cu} / \mathrm{NCQDs}$ have no dependence on $\mathrm{pH}$. To further measure the photobleaching resistance of the Cu/NCQDs, the same amount of the $\mathrm{Cu} / \mathrm{NCQD}$ solution was irradiated under a $360 \mathrm{~nm}$ UV lamp for $0,15,30,45,60,75,90,105$, and 120 min, after which its fluorescence emission spectrum was measured. As shown in Figure 6-C, the Cu/NCQDs dissolved as the duration of UV irradiation increased. The fluorescence intensity of the solution barely changed, indicating the excellent photobleaching resistance of the prepared $\mathrm{Cu} / \mathrm{NCQDs}$, which is essential for their practical application.

\subsection{Selective detection of metronidazole using Cu/NCQDs}

To evaluate the specificity of this method for metronidazole detection using $\mathrm{Cu} / \mathrm{NCQDs}$, the common potential interference substances in metronidazole drugs were studied. The $\mathrm{Cu} / \mathrm{NCQD}$ solution $(100 \mu \mathrm{L}), 2$ $\mathrm{mL}$ of the citric acid-sodium dihydrogen phosphate buffer $(\mathrm{pH}=4)$, and the metronidazole solution (100 $\mu \mathrm{mol} / \mathrm{L}$ ) were added into a series of $10 \mathrm{~mL}$-colorimetric tubes. Thereafter, the interference substances (200 mmol/L): $\mathrm{K}^{+}, \mathrm{Mg}^{2+}, \mathrm{NH}_{4}{ }^{+}, \mathrm{Ca}^{2+}, \mathrm{O}_{3}{ }^{2-}$, glucose, ascorbic acid, and sucrose, as well as $\beta$-cyclodextrin and L-cysteine $(5 \mathrm{mmol} / \mathrm{L})$, were added. The fluorescence intensity was recorded using a fluorescence spectrometer. As shown in Figure 7, the fluorescence intensities of the solutions with interfering substances (50 times and 200 times) and without interfering substances barely changed. The results validated the excellent specificity of the $\mathrm{Cu} / \mathrm{NCQD}$ fluorescence probe for metronidazole detection.

\subsection{Interaction mechanism between the Cu/NCQDs and metronidazole}

The interaction between the Cu/NCQDs and metronidazole is shown in Figure 8-A. As can be observed, metronidazole exerts a fluorescence quenching effect on the Cu/NCQDs, leading to a decrease in their fluorescence intensity. Therefore, the variation in the fluorescence intensity can be utilized as an indicator of the change in the concentration of metronidazole. Based on this, an on-off fluorescence-detection method using $\mathrm{Cu} / \mathrm{NCQDs}$ as the fluorescence probe was established for the determination of the metronidazole content. 
Figure 8-A shows that after the addition of metronidazole, the fluorescence emission peak of the Cu/NCQDs did not shift from $443 \mathrm{~nm}$, neither did a new emission peak appear, indicating that the quenching effect of metronidazole on the Cu/NCQDs is independent of the fluorescence resonance energy transfer. To further understand the quenching mechanism of metronidazole on the $\mathrm{Cu} / \mathrm{NCQD}$ fluorescence, the UV-vis absorption spectrum of metronidazole was compared with the fluorescence excitation spectrum of the Cu/NCQDs (Figure 8-B). In Figure 8-B, metronidazole displays two absorption bands at $\sim 230 \mathrm{~nm}$ and $318 \mathrm{~nm}$, while the maximum excitation wave peak in the Cu/NCQD spectrum is located at $\sim 355 \mathrm{~nm}$. Evidently, the absorption spectrum of metronidazole overlaps with the maximum excitation spectrum of the $\mathrm{Cu} / \mathrm{NCQD}$ s. Therefore, it can be concluded that the quenching effect of metronidazole on the Cu/NCQD fluorescence is related to the inner filter effect (IFE). In the detection system, metronidazole is the absorber, while the Cu/NCQDs are the fluorophore. Metronidazole absorbs the excitation energy of Cu/NCQDs, leading to a decrease in the fluorescence intensity of the Cu/NCQDs. To further determine the quenching mechanism of the system, MNZ and the mixed solution of MNZ and $\mathrm{Cu} / \mathrm{NCQD}$ s were subjected to UV-vis spectroscopy, as shown in Figure 8-C. After adding the Cu/NCQDs, the characteristic absorption peaks of MNZ did not change, and the electron-hole pair recombination did not occur, indicating that the quenching mechanism was unrelated to the photoinduced electron transfer process. Therefore, in this detection system, the quenching effect of metronidazole on the Cu/NCQDs can be explained by the IFE $[26,27]$.

\subsection{Analysis and test of actual samples}

The metronidazole-treated tablet solution $(20 \mu \mathrm{L}, 11.685 \mathrm{mmol} / \mathrm{L})$ was poured into a $10 \mathrm{~mL}$-colorimetric tube, and $100 \mu \mathrm{L}$ of the Cu/NCQD solution was added. Thereafter, the solution was diluted to scale with the citric acid-sodium dihydrogen phosphate buffer $(\mathrm{pH}=4)$, and its fluorescence intensity was measured using a fluorescence spectrophotometer. A portion of the honey-treatment solution was directly utilized for the fluorescence intensity measurement, and the labeled recovery experiment was conducted simultaneously. The experimental results are listed in Table 1. The calculated MNZ content in the metronidazole tablets was $0.1972 \mathrm{~g} /$ tablet, which was not significantly different from the theoretical value $(0.2 \mathrm{~g} /$ tablet $)$. The recovery rates of the metronidazole tablets and honey samples were $96.8-$ $103.7 \%$, and the relative standard deviation was lower than $6 \%$. The results showed the significant application potential of the fluorescence probe in detecting MNZ in actual samples. Compared with other fluorescence detection methods, the detection limit of this method is not the lowest, but the linear range is wider. The results are listed in Table 2. It is noteworthy that the fluorescent probe of this method uses natural biomass as carbon source and adopts one-step hydrothermal method, which is simpler and more environmentally friendly. 
Table 1

Determination results of residual MNZ in metronidazole effervescent tablets and honey

\begin{tabular}{|lllll|}
\hline Samples & Spiked $(\mu \mathrm{mol} / \mathrm{L})$ & $\begin{array}{l}\text { Total found } \\
(\mu \mathrm{mol} / \mathrm{L})\end{array}$ & $\begin{array}{l}\text { Recovery }(\%) \\
(\mathbf{n}=3)\end{array}$ & RSD (\%) \\
\hline MNZ & 0 & $23.02 \pm 0.21$ & - & - \\
\cline { 2 - 5 } & 20.00 & $42.26 \pm 0.24$ & 98.2 & 4.3 \\
\hline Commercially available honey & 0 & $65.37 \pm 0.46$ & 103.7 & 3.6 \\
\cline { 2 - 5 } & 20.00 & Not Found & - & - \\
\cline { 2 - 5 } & 40.00 & $19.35 \pm 0.53$ & 96.8 & 5.2 \\
\hline
\end{tabular}

Table2 summarizes the reported fluorescence-detection method of metronidazole compared with other fluorescence-detection methods.

\begin{tabular}{|c|c|c|c|c|c|}
\hline $\begin{array}{l}\text { Fluorescent } \\
\text { probe }\end{array}$ & Synthetic methods & $\begin{array}{l}\text { Carbon } \\
\text { source }\end{array}$ & $\begin{array}{l}\text { Linear range } \\
\text { ( } \mu \mathrm{mol} / \mathrm{L})\end{array}$ & $\begin{array}{l}\text { Detection limit } \\
(\mu \mathrm{mol} / \mathrm{L})\end{array}$ & Ref. \\
\hline $\mathrm{PCDs}^{\mathrm{a}}$ & $\begin{array}{l}\text { Thermal } \\
\text { decomposition } \\
\text { method }\end{array}$ & $\begin{array}{l}\text { Polyvinyl } \\
\text { imine }\end{array}$ & $0.3504-87.6$ & 0.12 & [28] \\
\hline $\begin{array}{l}\text { GQDs- } \\
\text { embedded } \\
\text { SMIPb }^{\mathrm{b}}\end{array}$ & & & $0.2-15$ & 0.15 & [29] \\
\hline $\begin{array}{l}\mathrm{g}-\mathrm{C}_{3} \mathrm{~N}_{4} \\
\text { nanosheets }\end{array}$ & $\begin{array}{l}\text { Thermal } \\
\text { polymerization }\end{array}$ & & $0.058-0.58$ & 0.05 & [30] \\
\hline CDs & Solvent hot method & $\begin{array}{l}\text { Acid } \\
\text { magenta6B } \\
\mathrm{H}_{2} \mathrm{O}_{2}\end{array}$ & $0-58.4$ & 1.50 & [27] \\
\hline FCNs & Hydrothermal method & gardenia & $0.8-225$ & 0.28 & [31] \\
\hline CDs & Solvent hot method & H. cordata & $3.3-240$ & 0.12 & [32] \\
\hline $\mathrm{Cu} / \mathrm{NCQDs}$ & Hydrothermal method & frangipani & $0-350$ & 0.28 & work \\
\hline
\end{tabular}

\section{Conclusion}

Blue fluorescent $\mathrm{Cu} / \mathrm{NCQD}$ s were successfully synthesized by a simple and green one-step hydrothermal method using frangipani as the carbon source. By introducing $\mathrm{Cu}$ and $\mathrm{N}$ diheteroatoms, the surface- 
energy trap of the Cu/NCQDs was effectively passivated by nitrogen-containing groups and Cucontaining groups $(\mathrm{N}-\mathrm{Cu}-\mathrm{N})$, leading to a non-excitation dependent fluorescence behavior. Further analysis of $\mathrm{Cu} / \mathrm{NCQD}$ s showed that the high-fluorescence intensity was stable and long-lasting, and the fluorescence emission was not affected by $\mathrm{pH}$.Therefore, a fluorescence-detection method using $\mathrm{Cu} / \mathrm{NCQDs}$ as an effective fluorescence probe to determine the metronidazole content in the range of $0-$ $350 \mathrm{~mol} / \mathrm{L}$ was established. The $\mathrm{R}^{2}$ value was 0.996 , and the detection limit of the method was 0.28 $\mathrm{mol} / \mathrm{L}$. This method was applied to analyze actual samples of medicine tablets and honey, and the results validated its potential for practical application. This method is expected to be advanced into a fluorescence kit for future practical analyses.

\section{Declarations}

\section{Acknowledgments}

This research was supported by Opening project of Guangxi Key Laboratory of Calcium Carbonate Resources Comprehensive Utilization No. HZXYKFKT201907.

\section{References}

[1]Jamie Katy Hu,Katherine Given Ligtenberg,Jonathan Leventhal,Christopher G. Bunick. Successful management of malodor from fungating tumors using crushed metronidazole tablets[J]. JAAD Case Reports,2020,6(1)

[2]Abdali Khadijeh,Jahed Leila,Amooee Sedigheh,Zarshenas Mahnaz,Tabatabaee Hamidreza,Bekhradi Reza. Comparison of the Effect of Vaginal Zataria multiflora Cream and Oral Metronidazole Pill on Results of Treatments for Vaginal Infections including Trichomoniasis and Bacterial Vaginosis in Women of Reproductive Age.[J]. BioMed research international,2015,2015:

[3]Kadhim M. Haddao. Investigation of metronidazole induced liver injury during early pregnancy in rats: A histological and histopathological studies[J]. Indian Journal of Public Health Research \& Development,2018,9(10):

[4] Xu H. Synthesis of fluorescent carbon quantum dots and its application in analysis and detection [D]. Nanchong: China West Normal University, 2016.

[5] Bilici M, Zengin A, Ekmen E, et al. Efficient and selective separation of metronidazole from human serum by using molecularly imprinted magnetic nanoparticles[J]. Journal of Separation Science, 2018, 41: $2952-2960$

[6] Zhang J. Determination of the content of metronidazole fenbufen capsules by HPLC [J]. Journal of Harbin Medical University, 2019, 32(4): 758-763. 
[7] Yang Chunmei. Simultaneous determination of metronidazole and tetracaine hydrochloride in Metronidazole coating by HPLC [J]. Clinical Rational Drug Use, 2019, 12(2): 118-119

[8] Zhou Yibing, Wu Kun, Li Lei, et al. Determination of metronidazole in honey by ULTRA-performance liquid chromatography-tandem mass spectrometry [J]. Chinese Journal of Physical and Chemical Detection, 2017, 53(8): 946-949

[9] Gao Hegang, Du Sai, Wang Rui, et al. Determination of chloramphenicol and metronidazole residues in honey by ULTRA performance liquid chromatography-tandem mass spectrometry [J]. Journal of Preventive Medicine, 2017, 29(9): 969-972

[10] Lei H Y, Guo J B, Lv Z, et al. Simultaneous determination of nitroimidazoles and quinolones in honey by modified QuEChERS and LC-MS/MS Analysis[J]. International Journal of Analytical Chemistry, 2018, 2018: 1-12

[11] Ma Xionghui, Wei Liuge, Wu Ting, et al. Journal of Analytical Measurement, 2017, 36(1): 91-95.

[12] Li Meina, LIANG Wenhui, Zhou Qing. Determination of metronidazole in toner by carbon paste electrode modified with multi-wall carbon nanotubes [J]. Chemical Analytical Metrology, 2019, 28(2): 5460

[13] Yan Zhengyu, Xiao An, Lv Hua, et al. Novel Carbon Materials, 2014, 29(3): 216-224.

[14] Sun Y P, Zhou B, Lin Y, et al. Quantum-Sized Carbon Dots for Bright and Colorful Photoluminescence[J]. Journal of the American Chemical Society, 2006, 128(24): 7756-7757

[15] Demchenko A P, Dekaliuk M O. Novel fluorescent carbonic nanomaterials for sensing and imaging[J]. Methods Appl Fluoresc, 2013, 1(4): 042001-042018

[16] Dong Y Q, Pang H C, Yang H B, et al. Carbon-based dots co-doped with nitrogen and sulfur for high quantum yield and excitation-independent emission[J]. Angewandte Chemie, 2013, 52(30): 7800-7804

[17]Dawei Fan,Xin Liu,Xinrong Shao,Yong Zhang,Nuo Zhang,Xueying Wang,Qin Wei,Huangxian Ju. A cardiac troponin I photoelectrochemical immunosensor: nitrogen-doped carbon quantum dots-bismuth oxyiodide-flower-like SnO2[J]. Microchimica Acta: Analytical Sciences Based on Micro- and Nanomaterials,2020,187(6):

[18]Li Quan. One-pot synthesis of 2,2'-dipicolylamine derived highly photoluminescent nitrogen-doped carbon quantum dots for Fe3+ detection and fingermark detection.[J]. Nanotechnology,2020,31(33):

[19] Zhang You. Preparation and application of metal/nonmetal doped carbon quantum dots [D]. Hebei University of Science and Technology,2019 
[20] SU R G. Preparation mechanism of metal doped carbon spot and application of heavy metal detection [D]. China University of Petroleum, Beijing,2018.

[21] Liu Y S, Wu P, Wu XY, et al. Nitrogen and copper(II) co-doped carbon dots for applications in ascorbic acid determination by non-oxidation reduction strategy and cellular imaging[J]. Talanta, 2020, 210: 120649-120658

[22] Ma Y S, Cen Y, Sohail M, et al. A ratiometric fluorescence universal platform based on N,Cu co-doped carbon dots to detect metabolitesparticipating in $\mathrm{H} 2 \mathrm{O} 2$-generation reactions[J]. ACS Applied Materials \& Interfaces, 2017, 9(38): 33011-33019

[23] An X T, Zhuo S J, Zhang P, et al. Carbon dots based turn-on fluorescent probes for oxytetracycline hydrochloride sensing[J]. RSC Advances, 2015, 5(26): 19853-19858

[24] Lin L P, Luo Y X, Tsai P Y, et al. Metal ions doped carbon quantum dots: Synthesis, physicochemical properties, and their applications[J]. Trends in Analytical Chemistry, 2018, 103: 87-101

[25] Dong Y Q, Shao J W, Chen C Q, et al. Blue luminescent graphene quantum dots and graphene oxide prepared by tuning the carbonization degree of citric acid[J]. Carbon, 2012, 50(12): 4738-4743

[26] Luo T, Bu L L, Peng S Y, et al. One-step microwave-assisted preparation of oxygen-rich multifunctional carbon quantum dots and their application for Cu2+-curcumin detection[J]. Talanta, 2019, 205: 1-9

[27] Zhao J R, Pan X H, Sun X B, et al. Detection of metronidazole in honey and metronidazole tablets using carbon dots-based sensor via the inner filter effect[J]. Luminescence, 2018, 33(4): 704-712

[28] Yang S L, Wang L, Zuo L, et al. Non-conjugated polymer carbon dots for fluorometric determination of metronidazole[J]. Microchimica Acta, 2019, 186(9): 652-661

[29] Mehrzad-Samarin M, Faridbod F, Dezfuli A S, et al. A novel metronidazole fluorescent nanosensor based on graphene quantum dots embedded silica molecularly imprinted polymer[J]. Biosensors and Bioelectronics, 2017, 92: 618-623

[30] Hatamie A, Marahel F, Sharifat A. Green synthesis of graphitic carbon nitride nanosheet (g-C3N4) and using it as a label-free fluorosensor for detection of metronidazole via quenching of the fluorescence[J]. Talanta, 2018, 176: 518-525

[31] Yang X P, Liu M X, Yin Y R, et al. Green, hydrothermal synthesis of fluorescent carbon nanodots from gardenia, enabling the detection of metronidazole in pharmaceuticals and rabbit plasma[J]. sensors, 2018, 18(4): 964-979

[32] Tang J H, Zhang Y H, Liu Y H, et al. Carbon quantum dots as a fluorophore for "inner filter effect" detection of metronidazole in pharmaceutical preparations[J]. RSC Advances, 2019, 9(65): 3817438182 
[33] Wu W T, Zhan L Y, Fan W Y, et al. Cu-N dopants boost electron transfer and photooxidation reactions of carbon dots[J]. Angewandte Chemie International Edition, 2015, 54(22): 6540-6544

[34] Wan $Y Y$, Wang $M$, Zhang $K L$, et al. Facile and green synthesis of fluorescent carbon dots from the flowers of Abelmoschus manihot (Linn.) Medicus for sensitive detection of 2,4,6-trinitrophenol and cellular imaging[J]. Microchemical Journal, 2019, 148: 385-396

[35] Li C J, Wang Y Q, Zhang X J, et al. Red fluorescent carbon dots with phenylboronic acid tags for quick detection of $\mathrm{Fe}(\mathrm{III})$ in PC12 cells[J]. Journal of Colloid and Interface Science, 2018, 526: 487-496

\section{Figures}

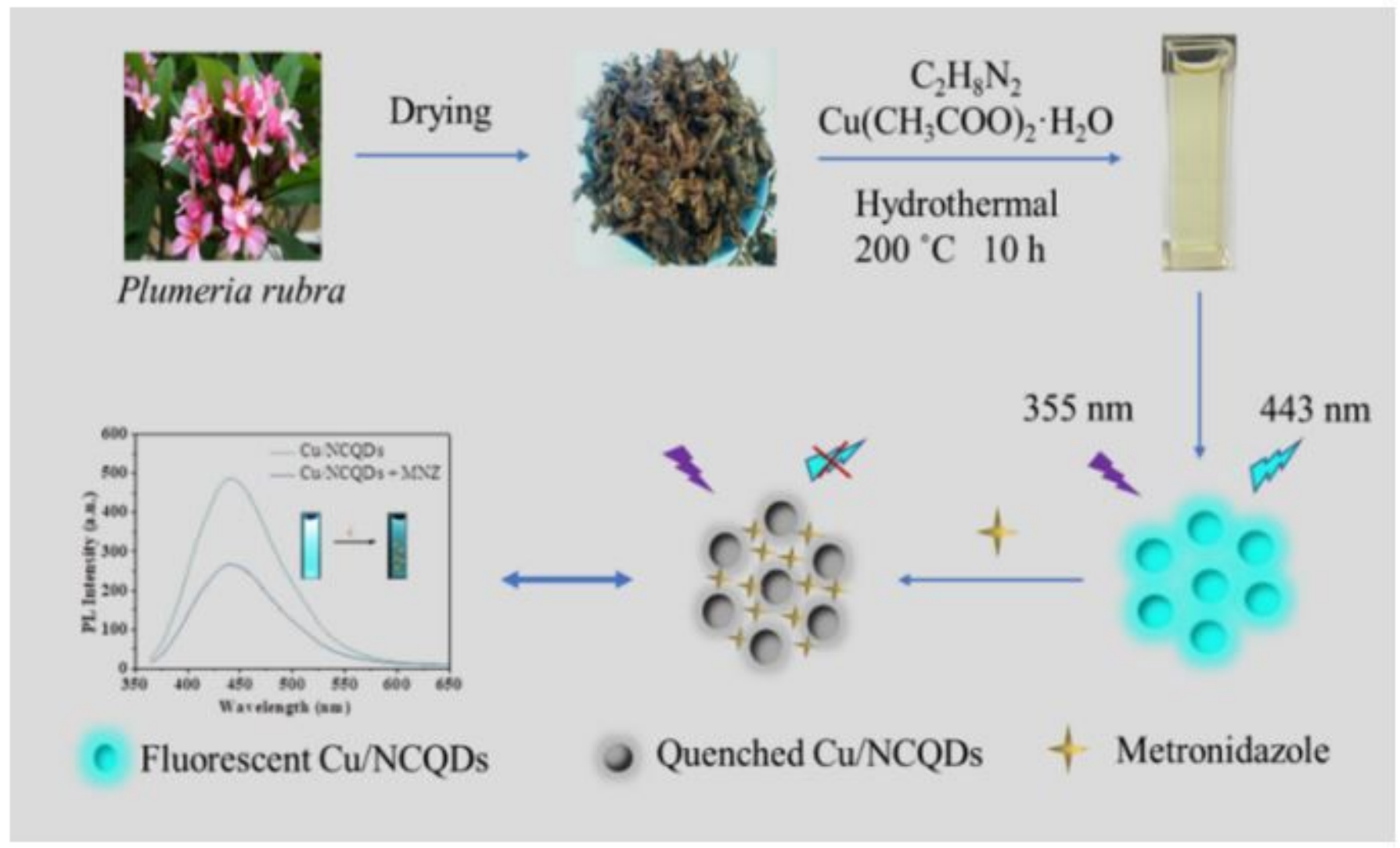

\section{Figure 1}

Schematic diagram of preparation of Cu/NCQDs and fluorescence detection of metronidazole 

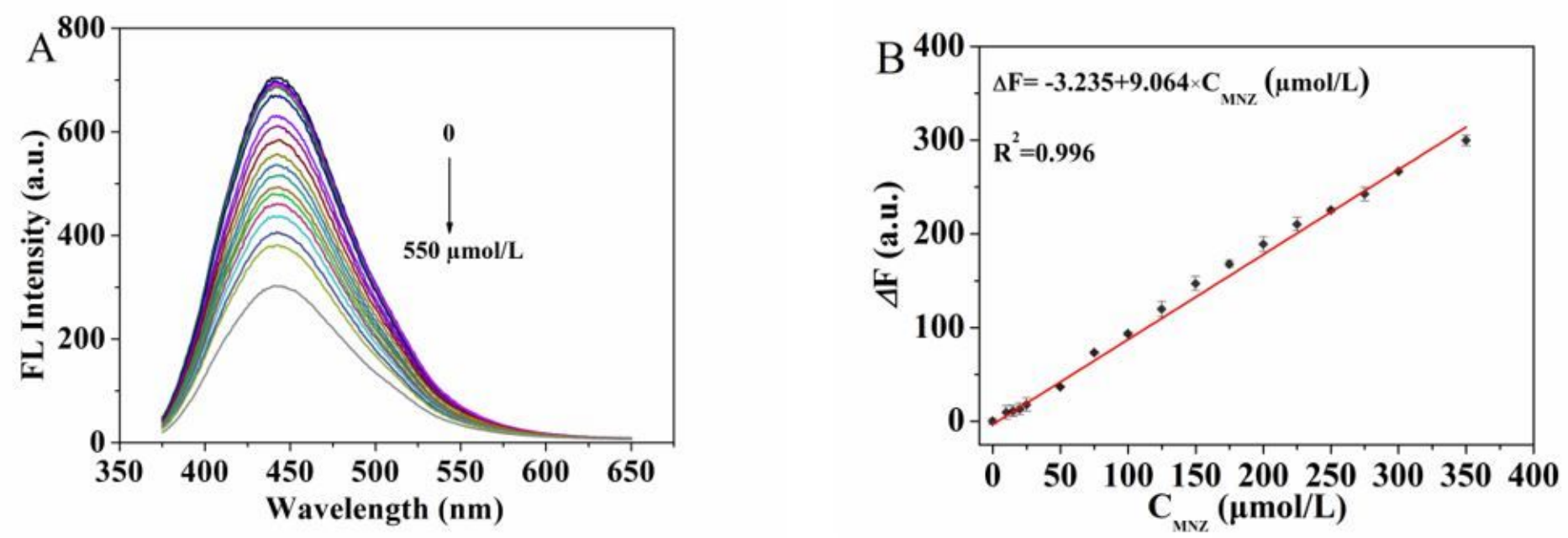

Figure 2

Fluorescence emission spectra of $\mathrm{Cu} / \mathrm{NCDs}-\mathrm{MNZ}$ system with different concentrations (A); The corresponding calibration curve (B) 

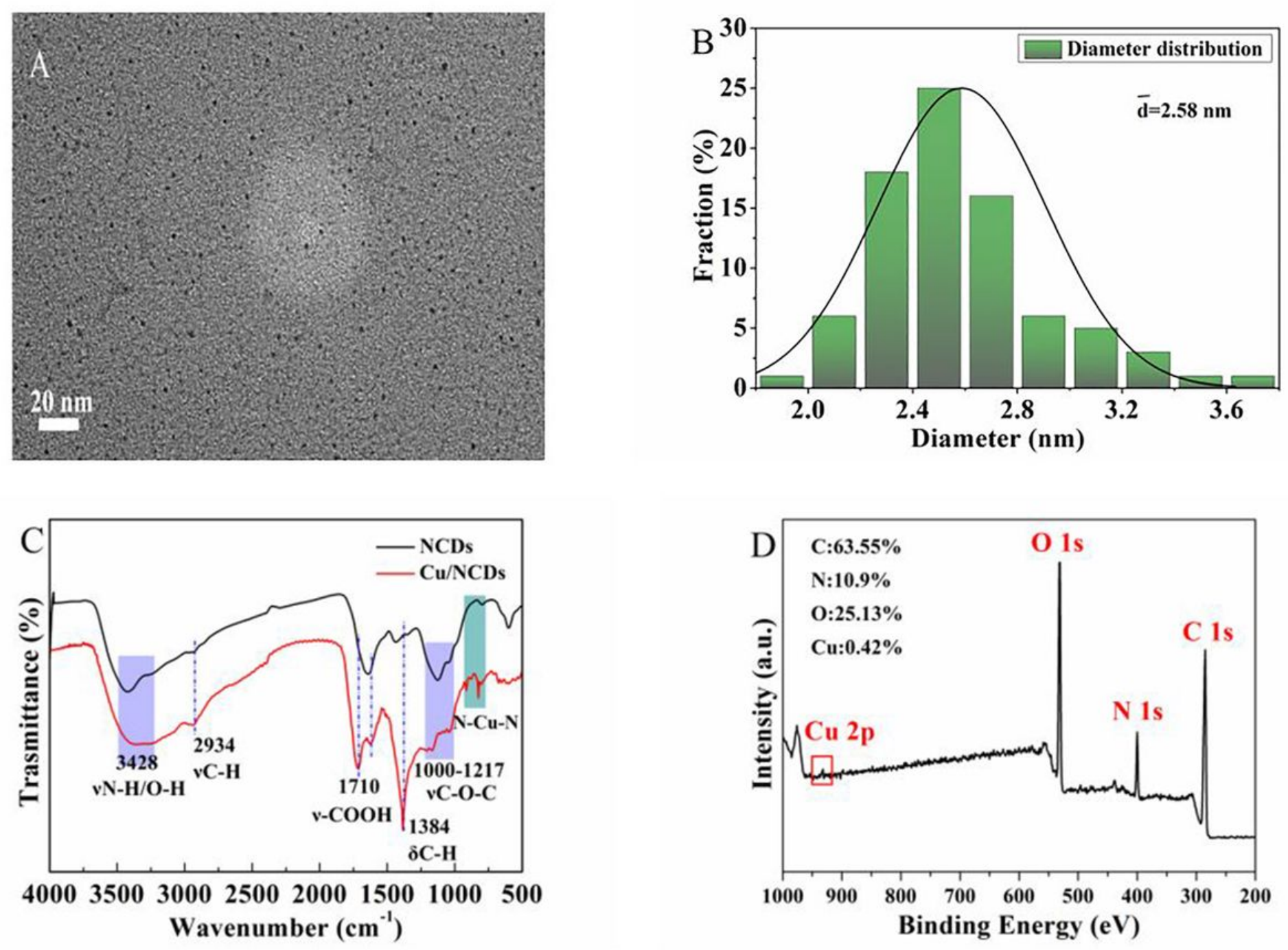

Figure 3

TEM image (A); particle size distribution image (B); FT-IR spectra (C); XPS full scan spectrum (D) of $\mathrm{Cu} / \mathrm{NCQDS}$ 

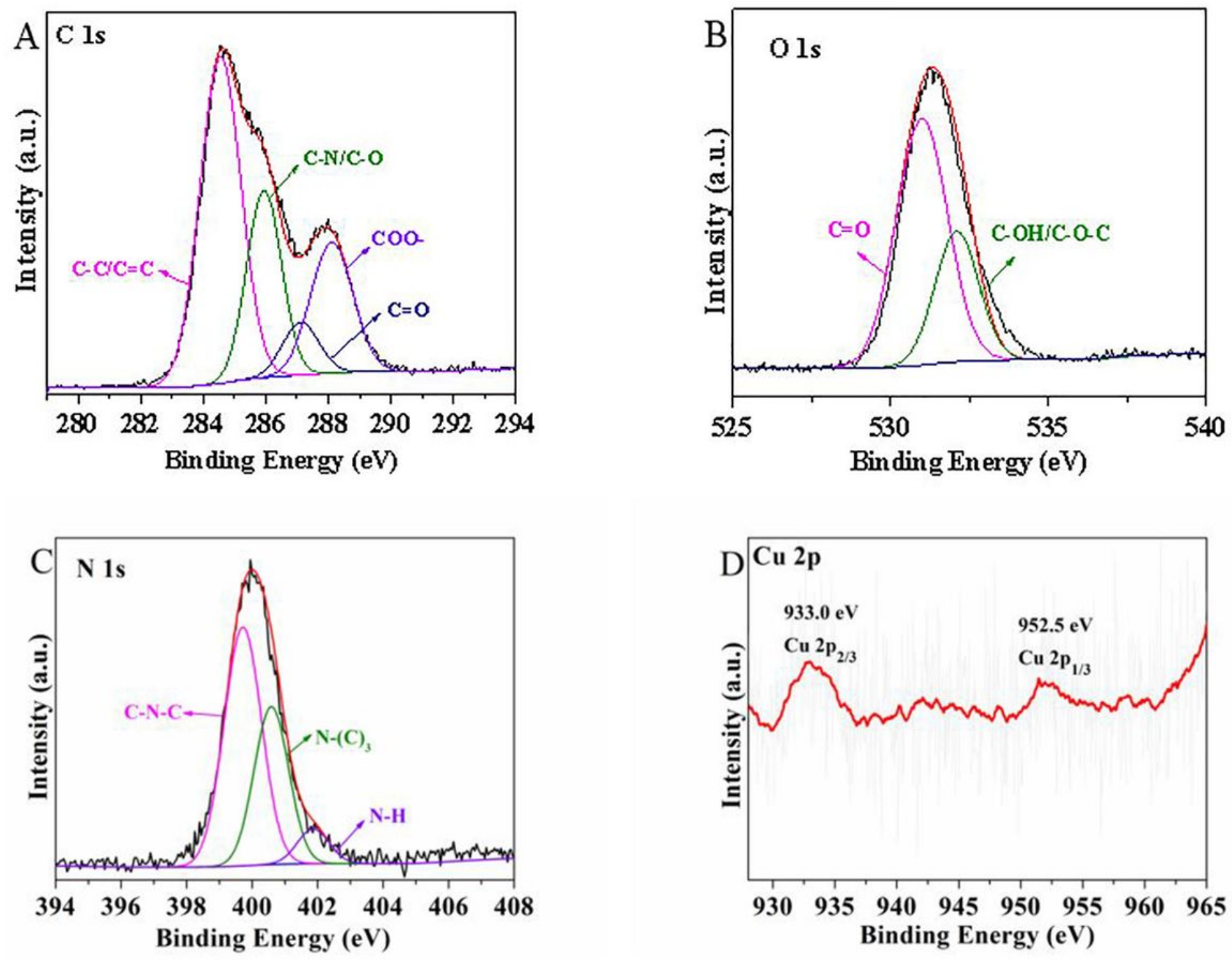

Figure 4

high-resolution XPS spectra of Cu/NCQDs: C 1s (A); 0 1s (B); N 1s (C) and Cu 2p (D) 

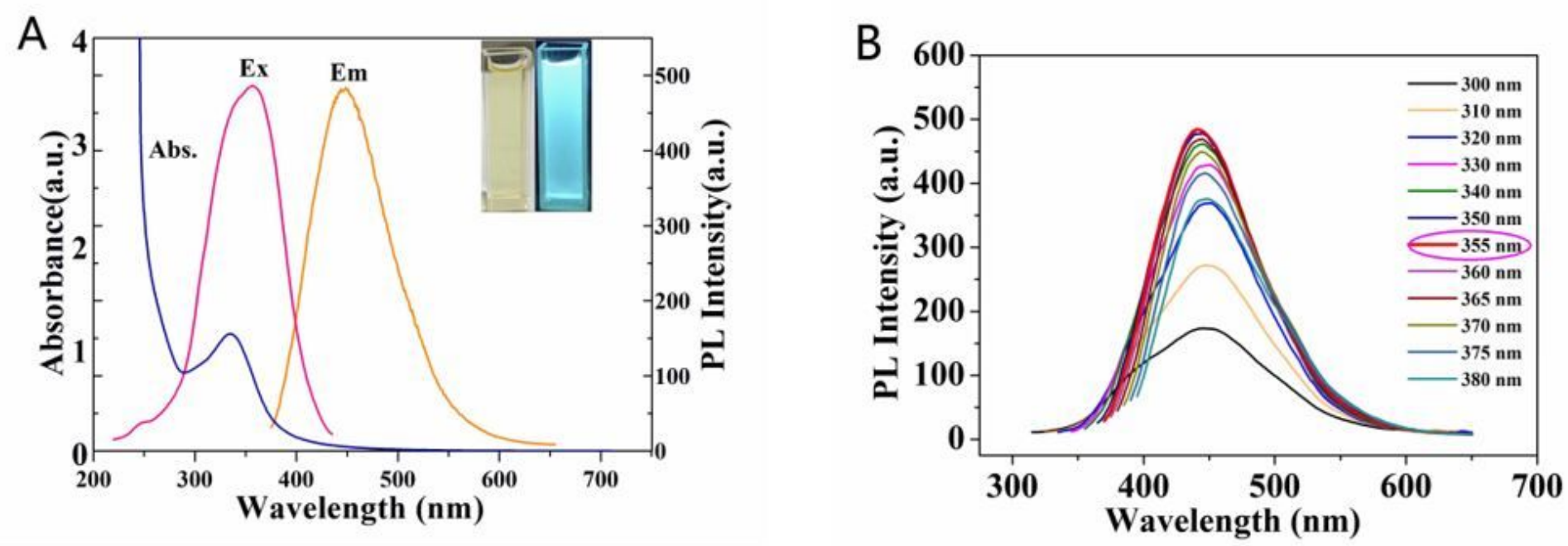

Figure 5

(A)UV-Vis spectrum, fluorescence excitation and emission spectra of Cu/NCQDs (Insets: visual images of $\mathrm{Cu} /$ NCQDs under daylight (left) and UV irradiation (right) at $360 \mathrm{~nm}$ ). (B)Fluorescence emission spectrum of $\mathrm{Cu} / \mathrm{NCQDs}$ at different excitation wavelengths 

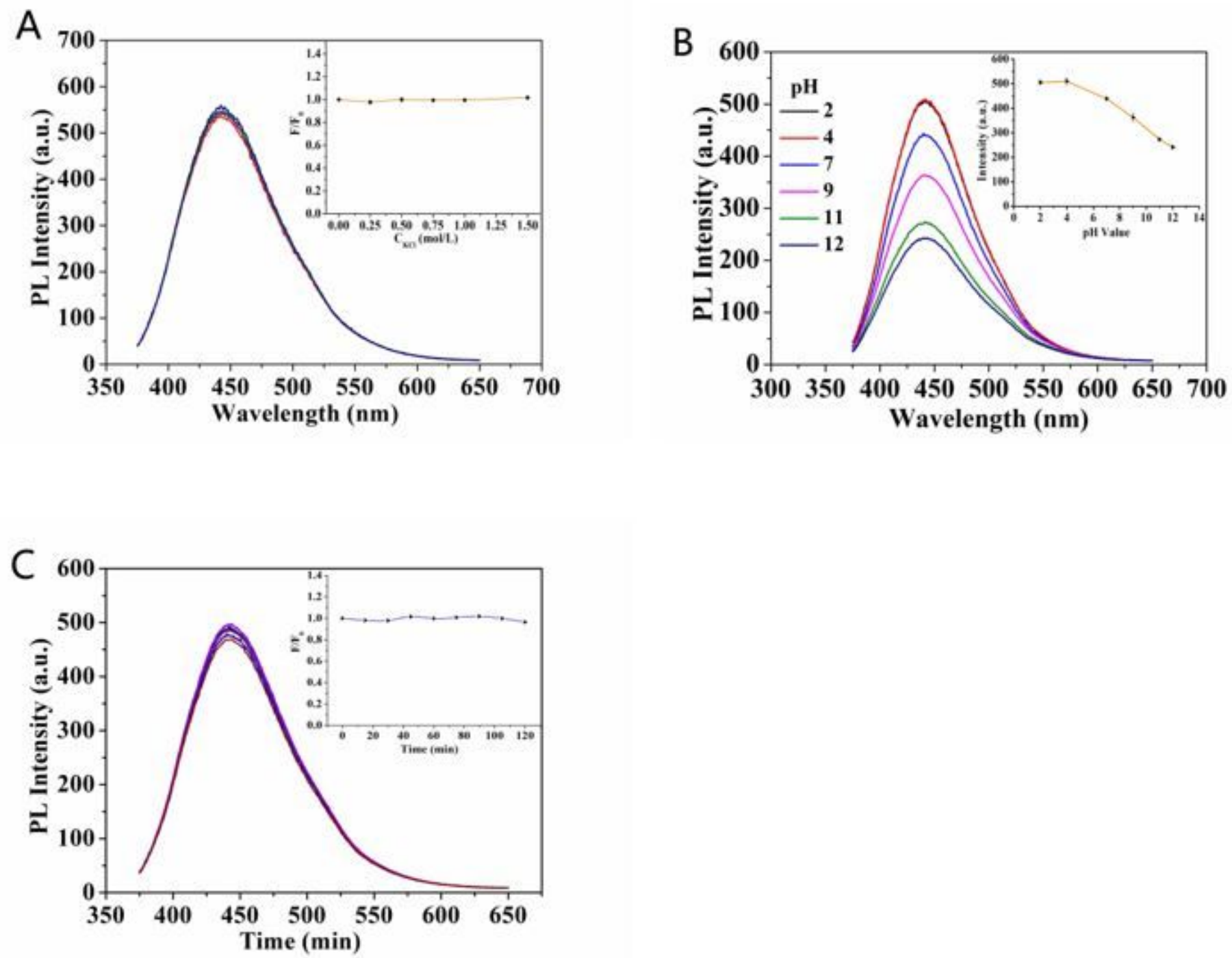

Figure 6

(A)Effects of different concentrations of $\mathrm{KCl}$ solution on the fluorescence properties of $\mathrm{Cu} / \mathrm{NCQDs}$.

(B)Fluorescence emission spectra of $\mathrm{Cu} / \mathrm{N}-\mathrm{CQD}$ s at different $\mathrm{pH}$ values; Inset: fluorescence emission intensity at different $\mathrm{pH}$ values. (C)Fluorescence emission spectrum of $\mathrm{Cu} / \mathrm{NCQDs}$ under different irradiation time by UV lamp; Inset: Fluorescence intensity of UV lamp irradiation at different time 


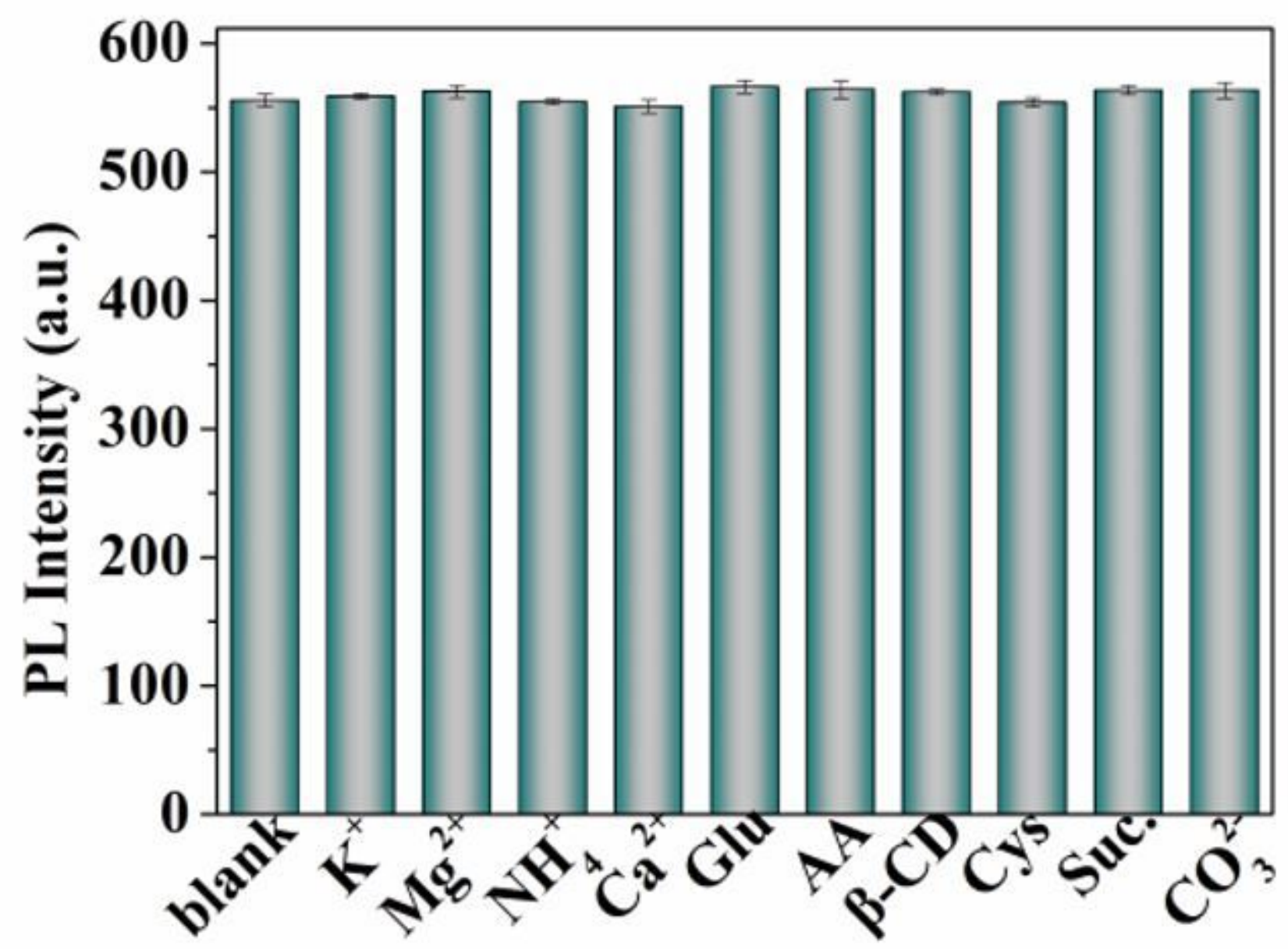

Figure 7

Effects of different potential interfering substances on the detection system of Cu/NCQDs-MNZ 

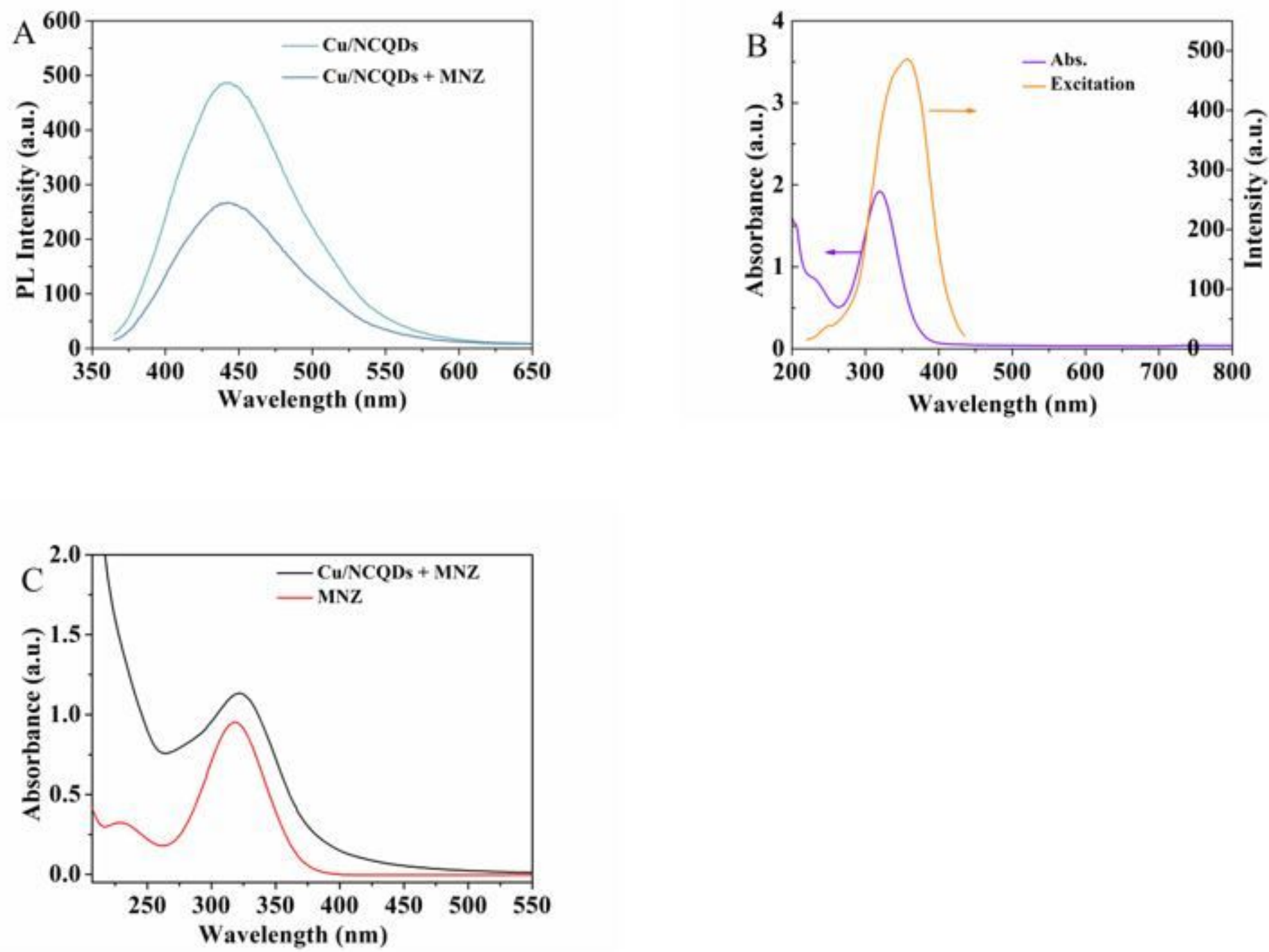

Figure 8

Fluorescence emission spectra of Cu/NCQDs with or without MNZ (A); The excitation spectra of $\mathrm{Cu} / \mathrm{NCQD}$ s and the UV-vis absorption spectra of MNZ (B); UV-vis absorption spectra of MNZ and $\mathrm{Cu} / \mathrm{NCQDs}$ mixed with MNZ (C) 